DOI:10.2478/rrlm-2019-0016

\title{
Is there any association between Serum anti-HSP27 antibody level and the presence of metabolic syndrome; population based case-control study
}

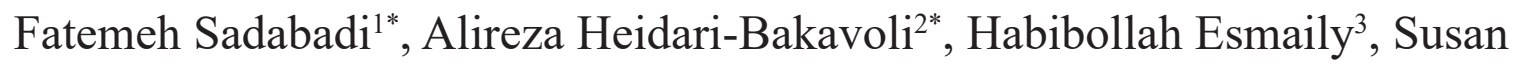
Darroudi ${ }^{1}$, Maryam Tayefi ${ }^{4}$, Zahra Asadi ${ }^{5}$, Seyed Mohammad Reza Parizadeh ${ }^{6}$, Shima Tavalaie ${ }^{6}$, Najmeh malekzadeh ${ }^{1}$, Kiana Hosseinpour Moghaddam ${ }^{7}$, Azam Rastgar Moghadam ${ }^{1}$, Amir Hosein Sahebkar ${ }^{8}$, Narges Fereydouni ${ }^{1}$, Elham Barati $^{1}$, Mahmoudreza Azarpazhooh ${ }^{2}$, Seyyed Javad Hosseini ${ }^{8}$, Mohammad Tayyebi², Mahmoud Ebrahimi², Gordon A. Ferns ${ }^{9}$, Majid Ghayour-Mobarhan ${ }^{5,6^{* *}}$, Mohsen Mouhebati ${ }^{2 * *}$

1. "Department of modern sciences and technologies, Faculty of medicine, Mashhad university of medical sciences, Mashhad, Iran

2. Cardiovascular Research Center, Faculty of Medicine, Mashhad University of Medical Sciences, Mashhad, Iran

3. Department of Biostatistics \& Epidemiology, Faculty of Health, Management \& Social Determinants of Health Research Center, Mashhad University of Medical Sciences, Mashhad, Iran

4. Clinical Research unit, Mashhad University of Medical Sciences, Mashhad, Iran

5. Department of Nutrition, Faculty of Medicine, Mashhad University of Medical Sciences, Mashhad, Iran

6. Metabolic Syndrome Research Center, Faculty of Medicine, Mashhad University of Medical Sciences, Mashhad, Iran

7. Department of biology, Faculty of sciences, Ferdowsi University of Mashhad, Mashhad, Iran

8. Department of Medical Biotechnology, Faculty of medicine, Mashhad University of medical sciences, Mashhad, Iran

9. Brighton \& Sussex Medical School, Division of Medical Education,

Falmer, Brighton, Sussex, UK

\footnotetext{
* Equally contributed as first authors; ** Equally contributed as Corresponding authors

Corresponding authors: Majid Ghayour-Mobarhan, MD, PhD; Biochemistry of Nutrition Research Center, Faculty of Medicine, Mashhad University of Medical Science, Mashhad, Iran, Postal code: 99199-91766; Email: ghayourm@mums.ac.ir.

Mohsen Mouhebati, MD; Cardiovascular Research Center, Faculty of Medicine, Mashhad University of Medical Sciences, Mashhad, Iran. Postal code: 99199-91766; Email: Mouhebatim@mums.ac.ir.
} 


\begin{abstract}
Background: Heat shock protein 27 (HSP27) is an intracellular chaperone constitutively expressed in many cell types including cardio myocytes and endothelial cells. Circulating levels of HSP27 and anti-HSP27 antibody are higher in patients with CVD. Anti-HSP27 antibody concentrations were also reported to be increased in atherogenesis. We aimed to evaluate serum anti-HSP27 antibody titers in individuals with, or without, MetS in the MASHAD study cohort with large sample size in 6,568 subjects. Methods: Participants with MetS were identified from MASHAD cohort ( $n=3358)$ using the IDF criteria, and the control group were those individuals who did not meet these criteria (n=3210). In-house enzyme-linked immune sorbent assay (ELISA) method was used for measuring Anti-HSP27 antibody levels. The two groups were matched for age, sex and smoking habit. Results: As expected, there were significant differences in height $(p=0.004)$, waist and hip circumference, weight, BMI, systolic and diastolic blood pressure, TGs, TC, HDL-C, Hs-CRP, glucose, with the presence of diabetes mellitus, hypertension, hyperlipidemia $(p<0.001)$ between the two groups. Serum HSP27 antibody titers did not show significant difference between the groups with and without metabolic syndrome $(p=0.740)$. Conclusion: In conclusion, our results revealed serum anti-HSP27 antibody titers were not statistically different between individuals with and without MetS. However, it is possible that drug treatment may affect antibody titers and confound our findings in this population sample. .
\end{abstract}

Keywords: serum anti-HSP27 antibody, Metabolic syndrome, MASHAD study cohort

Received: 15 th September 2018; Accepted: $9^{\text {th }}$ February 2019; Published: $15^{\text {th }}$ April 2019

\section{Introduction}

Metabolic syndrome (MetS), also called Reaven syndrome, or Syndrome X, is characterized by several risk factors of cardiovascular disease including central obesity, dyslipidemia, impaired glucose tolerance, high blood pressure (1-4), and a proinflammatory and prothrombotic state (5). MetS increases the risk of diabetes mellitus, coronary heart disease, stroke and other conditions including; fatty liver (3), asthma, ovarian cysts (4) and some cancers (6).

The prevalence of MetS varies from $<10 \%$ to $84 \%$ dependent on the definition used and population investigated (7). Genetic background, physical activity, diet, smoking, and family history of diabetes affect the risk of MetS (8). The prevalence of MetS is also affected by age; this increases with age group in most populations: $6.7 \%$ in the $20-29$ years and $43.5 \%$ in the $60-69$ years of age (9).
Heat shock proteins (HSPs) are intracellular proteins highly conserved, that have chaperonin activities, refolding denatured proteins. Several characteristic features of MetS (impaired glucose tolerance, hypertension, dyslipidemia) may be associated with oxidative stress that can induce the expression of HSPs $(10,11)$. HSP27 is a small HSP expressed and presented in a lot of cell types including endothelial cells and cardiomyocytes. The latter possess cardio-protective properties (12). Several studies have reported that the circulating levels of HSP27 and anti-HSP27 antibody are higher in CVD patients including acute coronary syndrome (ACS) and documented coronary artery disease (CAD) (13-15). Anti-HSP27 antibody concentrations were also reported to be increased in atherogenesis $(16,17)$. Since the response of immune system to HSPs may contribute to the development of atherosclerosis, the production of anti-HSP27 antibodies has been proposed to be a marker of inflammation (18), 
We aimed to evaluate serum anti-HSP27 antibody titers in individuals with, or without, MetS in the cohort study of Mashhad Stroke and Heart Atherosclerotic Disorder (MASHAD). Individuals were recruited into this study using the stratified cluster random sampling method. The cohort comprised 9,704 participants aged 35-65, who were selected from an urban population located within Northeastern Iran. The MASHAD study has been one of the first cohorts of CVD in the Middle East designed to estimate the incidence of cardiovascular diseases and assess the effect of different environmental, nutritional, psychosocial and genetic risk factors, started in 2007 and continued until 2020(19).

\section{Material and Methods}

\section{Subject}

This research was approved by the Mashhad University of Medical Sciences Ethics Committee in accordance with the Declaration of Helsinki (Ethical code no. IR.MUMS.REC.1386.250), Informed consent was obtained from all individuals recruited to the study (19). All participants with MetS were identified from MASHAD cohort $(n=3358)$ using the IDF criteria, and the control group were those individuals who did not meet these criteria $(n=3210)$.

\section{Study design}

The International Diabetes Federation (IDF) criteria, were: if three of the following five criteria were met: (1) abdominal obesity: waist circumference $>94 \mathrm{~cm}$ in men and $>80 \mathrm{~cm}$ in women; (2) hypertriglyceridemia $\geq 150 \mathrm{mg} / \mathrm{dl}$ or specific treatment; (3) serum HDL-C $<40 \mathrm{mg} / \mathrm{dl}$ in men and $<50 \mathrm{mg} / \mathrm{dl}$ in women or specific treatment; (4) blood pressure (HBP) $\geq 130 / 85 \mathrm{mmHg}$ or specific treatment; (5) high fasting glucose $\geq 100$ $\mathrm{mg} / \mathrm{dl}$ or treatment with anti-diabetic drugs(5). We excluded individuals who had CVD before entering the study and pregnant and breastfeed- ing women.Also full drug history was taken from each subject because of the probable potential confounding effects of drugs on Hsp27 antibody titers. Participants taking glucocorticoids, nonsteroidal anti-inflammatory (NSAIDs), anticoagulant drugs and psychiatric medications were excluded. A high proportion of patients with MetS were prescribed anti-diabetic drug, anti-hypertensive agent and statins and were not excluded from the study.

\section{Blood sampling and Biochemical measure- ments}

At the start of the study, a fasted blood sample (after 12 hours fast) was obtained from all participants. Anthropometric parameters, including: weight, height, waist circumference, hip circumference, body mass index (BMI), blood pressure, etc. were measured and recorded. Demographic data were collected using a questionnaire. Physical activity level (PAL) is the numerical variable expressing an individual's daily physical activity, assessed from James and Schofield human energy requirement equations.

Biochemical parameters measured included: fasting blood glucose (FBG), lipid profile including serum total cholesterol (TC), triglycerides (TGs), high density lipoprotein cholesterol (HDL-C) and serum hsCRP, and were measured using commercial kits and an Alycon auto analyzer (ABBOTT, Chicago, IL, USA). LDL-C (Low-density lipoprotein cholesterol) concentrations were estimated using the Friedewald formula (20).

\section{Measuring of anti-HSP27 antibody}

In-house enzyme-linked immune sorbent assay (ELISA) method was used for measuring Anti-HSP27 antibody levels. In brief, 50-ng recombinant human HSP27 antigen was added to 50 $\mu$ Lcarbonate buffer $\mathrm{pH}=9.6$ and then micro-titer plates (Nunc Maxisorp, Nunc) were coated. The plates were incubated at $4{ }^{\circ} \mathrm{C}$ under humid con- 
ditions for 18 hours. After this time, the wells were washed three times with buffer phosphate saline with $0.05 \%$ Tween-20 (PBST). For blocking stage and decreasing non-specific binding, wells were filled and incubated with $2 \%$ goat serum in PBS. The wells were incubated for 30 min in $37^{\circ} \mathrm{C}$ and $30 \mathrm{~min}$ at room temperature. After washing (three times with PBS), $100 \mu \mathrm{L}$ of diluted serum 1:100 with $2 \%$ goat serum in PBS was added to each well in duplicate and incubated $30 \mathrm{~min}$ at room temperature. Wells were washed six times (four times in PBST and two times in PBS). Then peroxide conjugated-goat anti-human IgG (Sigma-Aldrich, Poole, UK) diluted 1:500with 2\% goat serum in PBS. 100 $\mu \mathrm{L}$ of diluted anti-human IgG was placed in each well. In this step the incubation time was 30 min at room temperature. Finally, each well was washed six times (four times in PBST and two times in PBS).

The tetramethyl benzidine (TMB) substrate was prepared $[100 \mu \mathrm{L}$ of $6 \mathrm{mg} / \mathrm{mLTMB}$ in dimethyl sulfoxide (DMSO)] and $100 \mu \mathrm{L}$ of TMB and 3 $\mu \mathrm{H} 2 \mathrm{O} 2$ was added to $10 \mathrm{~mL}$ of acetate buffer, $50 \mathrm{mM}$ and $\mathrm{pH} 4.5$. Finally, $100 \mu \mathrm{L}$ of substrate solution was added per well and the plate was incubated for $15 \mathrm{~min}$ in the dark at room temperature. To terminate the reaction, $50 \mu \mathrm{L}$ of 3 $\mathrm{M} \mathrm{HCl}$ was added. The optical density of each well was evaluated at $450 \mathrm{~nm}$ using Lab systems iEMS Reader MF microtiter plate reader. The reference wavelength was $620 \mathrm{~nm}$.

\section{Statistical analysis}

SPSS version16 was used for all statistical analyses. Variables with normally and none normally distribution were expressed as means $\pm \mathrm{SD}$, median and interquartile range respectively. The groups were matched according to algorithms for pair-matching based on the propensity score by age, sex and smoking $(\mathrm{P}=0.231,0.798$ and 0.718 respectively) (21). Comparison of groups was accomplished by ANOVA, or Kruskal-Wal- lis tests, and a Bonferonni correction applied for multiple comparisons. In order to assess the independent effects of taking medication and PAL on MetS and its components on measures of serum anti-HSP27 antibody levels, multiple regression analyses were performed. The level of statistical significance was $\mathrm{p}<0.05$.

\section{Result}

Baseline anthropometric, demographic and biochemical characteristics between participants with MetS and controls are shown in Table 1. As expected, there were significant differences in height ( $p=0.004)$, weight, waist and hip circumference, BMI, systolic and diastolic blood pressure, TGs, TC, HDL-C, Hs-CRP, glucose, and the presence of diabetes mellitus, hypertension, hyperlipidemia and PAL between two groups $(p<0.001)$. There was not significant difference in serum HSP27 antibody titers and LDL-C between the two groups $(p=0.740$ and $p=0.694$ respectively).

There were significant differences between those with and without MetS in prescription for medication such as anti-diabetic drug, anti-hypertensive agent, statins $(\mathrm{p}<0.001)$.

Anti-HSP27 levels were compared in groups that were positive for different numbers of MetS criteria as showed in Table 2, there were no significant differences in serum anti-HSP27 titers with increasing components of MetS.

Different models of multivariate regression for anti-HSP27 and MetS were undertaken, and the effect of using drug and PAL on anti-HSP27 levels assessed (Table 3). In model 1 (unadjusted), model 2 (adjusted for taking anti-diabetic drug, anti-hypertensive agent and statins) and model 3 (adjusted for taking anti-diabetic drug, anti-hypertensive agent, statins and PAL), no significant relation between anti-HSP27 level and MetS was observed. 
Table1. Comparison of the baseline anthropometric, demographic and biochemical characteristics between participant with or without MetS

\begin{tabular}{|c|c|c|c|c|}
\hline \multicolumn{2}{|l|}{+2} & MetS $^{+}(\mathrm{n}=\mathbf{3 3 5 8})$ & MetS' $^{-}(n=3210)$ & p value \\
\hline \multicolumn{2}{|l|}{ Age (y) } & $49.43 \pm 7.94$ & $49.19 \pm 8.19$ & 0.231 \\
\hline \multirow{2}{*}{$\operatorname{Sex} N(\%)$} & Male & $1035(31.3)$ & $1015(31.6)$ & \multirow[t]{2}{*}{0.810} \\
\hline & Female & $2269(68.7)$ & $2195(68.4)$ & \\
\hline \multicolumn{2}{|l|}{ Height (m) } & $1.59 \pm 0.09$ & $1.58 \pm 0.08$ & 0.004 \\
\hline \multicolumn{2}{|l|}{ Weight (kg) } & $75.85 \pm 12.66$ & $68.92 \pm 12.64$ & $<0.001$ \\
\hline \multicolumn{2}{|c|}{ Waist circumference $(\mathrm{cm})$} & $100.40 \pm 10.19$ & $93.42 \pm 12.50$ & $<0.001$ \\
\hline \multicolumn{2}{|l|}{ Hip circumference (cm) } & $106.63 \pm 8.91$ & $102.72 \pm 9.57$ & $<0.001$ \\
\hline \multicolumn{2}{|l|}{ BMI (kg/m2) } & $29.78 \pm 4.33$ & $27.32 \pm 4.78$ & $<0.001$ \\
\hline \multicolumn{2}{|c|}{ Systolic blood pressure (mm Hg) } & $129.50 \pm 20.69$ & $118.46 \pm 16.06$ & $<0.001$ \\
\hline \multicolumn{2}{|c|}{ Diastolic blood pressure (mm Hg) } & $83.59 \pm 12.21$ & $76.91 \pm 10.83$ & $<0.001$ \\
\hline \multicolumn{2}{|c|}{ Triglycerides (mg/dl) } & $177(136-233)^{\mathrm{a}}$ & $100(75-128)^{\mathrm{a}}$ & $<0.001$ \\
\hline \multicolumn{2}{|l|}{ Total Cholesterol (mg/dl) } & $199.56 \pm 40.83$ & $189.90 \pm 38.40$ & $<0.001$ \\
\hline \multicolumn{2}{|l|}{ HDL-C(mg/dl) } & $39.11 \pm 7.67$ & $46.07 \pm 10.52$ & $<0.001$ \\
\hline \multicolumn{2}{|l|}{ LDL-C(mg/dl) } & $118.00 \pm 38.09$ & $118.35 \pm 34.43$ & 0.694 \\
\hline \multicolumn{2}{|l|}{ Glucose (mg/dl) } & $99.28 \pm 45.23$ & $90.43 \pm 37.46$ & $<0.001$ \\
\hline \multicolumn{2}{|l|}{ Hs-CRP (mg/dl) } & $2.03(1.20-4.16)^{\mathrm{a}}$ & $0.215(0.95-3.33)^{\mathrm{a}}$ & $<0.001$ \\
\hline \multicolumn{2}{|c|}{ HSP27 antibody titers (OD) } & $0.203(0.104-0.336)^{\mathrm{a}}$ & $0.202(0.105-0.335)^{\mathrm{a}}$ & 0.740 \\
\hline \multirow[t]{3}{*}{ Smoking habit, N (\%) } & Never & $2301(69.7)$ & $2229(69.4)$ & \multirow[t]{3}{*}{0.841} \\
\hline & Past & $318(9.6)$ & $300(9.3)$ & \\
\hline & Current & $684(20.7)$ & $681(21.2)$ & \\
\hline \multicolumn{2}{|c|}{ Diabetes mellitus N (\%) } & $644(19.7)$ & $393(12.4)$ & $<0.001$ \\
\hline \multicolumn{2}{|l|}{ Hypertension N (\%) } & $1646(50.2)$ & $763(24)$ & $<0.001$ \\
\hline \multicolumn{2}{|l|}{ Hyperlipidemia N (\%) } & $3257(98.6)$ & $2430(75.7)$ & $<0.001$ \\
\hline \multirow{4}{*}{ PAL } & Sedentary & $789(27)$ & $496(16.9)$ & \multirow[t]{4}{*}{$<0.001$} \\
\hline & Low active & $860(29.5)$ & $741(25.2)$ & \\
\hline & Moderately active & $1032(35.3)$ & $1231(41.8)$ & \\
\hline & High active & $239(8.2)$ & $475(16.1)$ & \\
\hline
\end{tabular}

BMI bodymass index, LDL-C low-density lipoprotein cholesterol, HDL-C high-density lipoprotein cholesterol, Hs-CRP highsensitivity C-reactive, aMedian (interquartile range) because of skewed distribution.

Serum HSP27 antibody titers were also compared according to the specific phenotypes of MetS shown in figure 1. Anti-HSP27 antibody titers were significantly higher in participants with waist circumstances criteria than subjects without any criteria of MetS $(\mathrm{p}<0.05)$ and patients with high waist circumstances, high density lipoprotein, triglycerides and blood pressure

Table 2. Serum HSP27 antibody concentration and number of MetS components

\begin{tabular}{lccccccc}
\hline \multicolumn{7}{c}{ Components number of metabolic syndrome } \\
\cline { 1 - 6 } & 0 & 1 & 2 & 3 & 4 & 5 & \multirow{2}{*}{ P-value } \\
\cline { 1 - 6 } Anti-HSP27 & $\mathbf{2 7 1}$ & $\mathbf{9 6 6}$ & $\mathbf{1 7 0 7}$ & $\mathbf{2 1 8 1}$ & $\mathbf{7 0 6}$ & $\mathbf{8 2}$ & \\
\multirow{2}{*}{ (OD) } & 0.184 & 0.193 & 0.207 & 0.207 & 0.190 & 0.216 & \\
& $(0.102-$ & $(0.103-$ & $(0.107-$ & $(0.105-$ & $(0.098-$ & $(0.108-$ & 0.570 \\
& $0.315)$ & $0.325)$ & $0.340)$ & $0.340)$ & $.324)$ & $0.319)$ & \\
\hline
\end{tabular}


Table 3. Different model of multivariate regression for anti-HSP27 and MetS

\begin{tabular}{lccc}
\hline & Model & OR (CI 95\%) & P value \\
\hline \multirow{3}{*}{ Anti-HSP27 (OD) } & Model 1 & $1.032(0.738-1.445)$ & 0.853 \\
\cline { 2 - 4 } & Model 2 & $0.954(0.738-1.448)$ & 0.848 \\
\cline { 2 - 4 } & Model 3 & $0.954(0.676-1.347)$ & 0.788 \\
\hline
\end{tabular}

Model 1: unadjusted; Model 2: adjusted for taking anti-diabetic drug, anti-hypertensive agent and statins; Model 3: adjusted for taking anti-diabetic drug, anti-hypertensive agent, statins and PAL. PAL: Physical Activity Level.

$(\mathrm{p}<0.01)$. Also there was a significant difference in serum HSP27 antibody titers between the subjects with high waist circumstances, high density lipoprotein, triglycerides and blood pressure compared to participanst with high waist circumstances, high density lipoprotein, blood pressure and glucose $(p=0.05)$.

\section{Discussion}

We have measured serum anti-HSP27 antibody titers in individuals with or without MetS recruited in the MASHAD study. Serum HSP27 antibody levels did not show significant difference between MetS positive (MetS+) and negative (MetS-) groups. The result of this study did not show additive effect in increasing MetS component on serum anti-HSP27 antibody titers. Previous studies have shown higher HSP antibody titers, release and consequently immune response to HSPs being affected by different MetS-related risk features such as diabetes, hypertension, hyperlipidemia and smoking which is most likely due to the induction of oxidative stress (22-24). Sahebkar et al. showed an increase in anti-HSP27 antibody levels in patients with coronary heart disease (CHD) and MetS compared to individuals with $\mathrm{CHD}$ alone (Sahebkar et al. 2011), which is in agreement with the study of Ghayour-Mobarhan et al. (25). Kargari et al. found that obese individuals showed significantly higher anti-HSP27 antibody levels than the non-obese group (26). However, they did not find any association between anti-HSP27

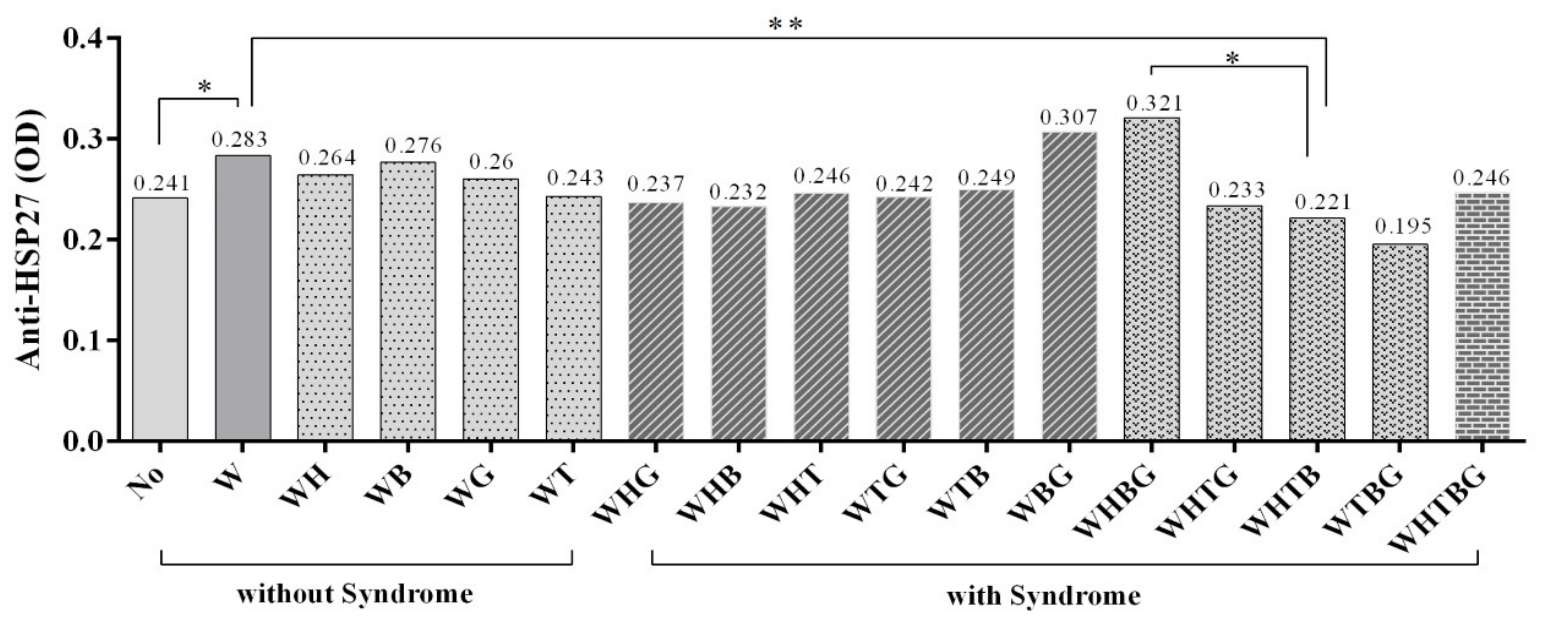

Fig. 1. Anti-HSP27 antibody according to phenotype of MetS

W: waist circumstances, H: high density lipoprotein, T: triglycerides, B: blood pressure, G: glucose ${ }^{*} \mathrm{p}<0.05 * * \mathrm{p}<0.01 ;{ }^{\mathrm{a}}$ Median (interquartile range) because of skewed distribution. 
antibody titers and either diabetes or MetS. They suggested that the previous results and inconsistencies may be because of the differences in the health status of the MetS- groups between the studies (2).

We measured anti-HSP antibody titers of MetS+ and MetS- individuals based on whether or not they had cardiovascular disease or diabetes. These results were non-significant either. We expect the reason for this misalignment of results may be due to the differences in the drugs taken by the two groups. Most people with MetS take cholesterol-lowering drugs (e.g. statins), blood glucose control drugs (e.g. insulin and metformin), blood pressure medications, etc. These drugs can affect the expression of HSPs.

Metformin, a metabolic stressor that potentially activates AMPK pathway, is a first-line medication to treat type 2 diabetes and it is consumed by hundred millions of people worldwide (27). Studies showed that metformin impairs the DNA-binding activity of HSF1 by the induction of ser121 phosphorylation through AMPK activation. Inhibition of HSF1 activity leads to down-regulation of HSPs (28). Statins can also cause a significant reduction in serum anti-HSPs levels. In our previous studies, we found that 40 $\mathrm{mg}$ /day statin therapy for 4 weeks was associated with decrease in anti-HSP antibody 60 and 70 titres in dyslipidemic patients $(29,30)$, while our next studies show that statin therapy did not significantly affect serum HSP60 and 70 (31). This phenomenon may be attributed to the immunomodulatory properties of statins. Since individuals with MetS often take metformin and statins because of hyperglycemia and dyslipidemia, decrease of anti-HSP27 antibody might be due to the use of these drugs in the group.

Another explanation for this result might be due to the removal of HSP27 by the immune system when it is released into the circulation. Burut et al. measured HSP27 IgG and IgM antibody levels between the CVD and non-CVD individuals with or without glucose tolerance. They showed there was not any significant difference in HSP27 IgG antibody between the groups but HSP27 IgM antibody levels were significantly lower in the normal glucose tolerance with CVD patients, compared with normal glucose tolerance without CVD patients. They suggested that the release of HSP27 IgM antibody into circulation may lead to formation of immune complexes. This may be one reason for the clearance of released HSP27 in patients with acute coronary syndromes (32). We did not evaluate Hsp27 IgM antibody titers in this study but found similar serum HSP27 IgG antibody levels in MetS+ and MetS- group. Serum anti Hsp27 antibody titers in two groups may be affected by the formation of antigen-antibody complexes and this might have happened to our HSP27 IgG antibody of MetS+ samples.

In conclusion, our results revealed that there was no significant difference in serum HSP27 antibody titers between MetS positive and negative groups.

This may be due to the effect of their use of administered drugs with MetS individuals. This result needs further knowledge about the effect of whole drugs consumed by MetS patients on HSP27 and anti-HSP27 antibody.

\section{Acknowledgment}

We thank all the patients and their family members for volunteering to participate in this study.

\section{Compliance with Ethical Standards}

This research was approved by the Mashhad University of Medical Sciences Ethics Committee in accordance with the Declaration of Helsinki (Ethical code no. IR.MUMS.REC.1386.250). Informed consent was obtained from all individuals recruited to the study. 


\section{Authors' contribution}

FS (Conceptualization; Data curation; Formal analysis; Software; Visualization; Writing original draft)

AHB (Conceptualization; Project administration; Visualization)

HE (Conceptualization; Formal analysis; Project administration; Software)

SD (Formal analysis; Investigation)

MT (Formal analysis; Software)

ZA (Data curation)

SMRP (Conceptualization; Writing - review \& editing)

ST (Methodology)

KHM (Investigation)

ARM (Conceptualization)

AHS (Conceptualization; Methodology)

NF (Conceptualization)

EB (Methodology)

MA (Investigation)

SJH (Visualization)

MT (Conceptualization)

ME (Conceptualization)

GAF (Writing - review \& editing)

MGM (Conceptualization; Project administration; Supervision)

MM (Conceptualization; Project administration; Supervision)

\section{Funding sources}

This work was supported by the Deputy of Research, Mashhad University of Medical Sciences [grant number 930940].

\section{Conflict of Interest}

The authors have no conflict of interest to disclose.

\section{References}

1. Takata H, Fujimoto S. Metabolic syndrome. Nihon rinsho Japanese journal of clinical medicine. 2013;71(2):266-9.

2. Kargari M, Parizadeh SMR, Karimian MS, Farahmand
SK, Sahebkar A, Esmaeili H, et al. Serum anti-HSP27 antibody titers in patients with metabolic syndrome, with or without diabetes mellitus. Comparative Clinical Pathology. 2016;25(4):895-901. DOI: 10.1007/s00580016-2279-0

3. Ford ES, Giles WH, Dietz WH. Prevalence of the metabolic syndrome among US adults: findings from the third National Health and Nutrition Examination Survey. Jama. 2002;287(3):356-9. DOI: 10.1001/ jama.287.3.356

4. Brumpton BM, Camargo CA, Romundstad PR, Langhammer A, Chen Y, Mai X-M. Metabolic syndrome and incidence of asthma in adults: the HUNT study. European Respiratory Journal. 2013;42(6):1495-502. DOI: 10.1183/09031936.00046013

5. Alberti KGM, Zimmet P, Shaw J. The metabolic syndrome - a new worldwide definition. The Lancet. 2005;366(9491):1059-62. DOI: $10.1016 / \mathrm{S} 0140-$ 6736(05)67402-8

6. Braun S, Bitton-Worms K, LeRoith D. The link between the metabolic syndrome and cancer. International journal of biological sciences. 2011;7(7):1003. DOI: 10.7150/ijbs.7.1003

7. van Vliet-Ostaptchouk JV, Nuotio M-L, Slagter SN, Doiron D, Fischer K, Foco L, et al. The prevalence of metabolic syndrome and metabolically healthy obesity in Europe: a collaborative analysis of ten large cohort studies. BMC endocrine disorders. 2014;14(1):9. DOI: 10.1186/1472-6823-14-9

8. Cameron AJ, Shaw JE, Zimmet PZ. The metabolic syndrome: prevalence in worldwide populations. Endocrinology and metabolism clinics of North America. 2004;33(2):351-75. DOI: 10.1016/j.ecl.2004.03.005

9. Miranda PJ, DeFronzo RA, Califf RM, Guyton JR. Metabolic syndrome: definition, pathophysiology, and mechanisms. American heart journal. 2005;149(1):3345. DOI: 10.1016/j.ahj.2004.07.013

10. Radons J. The Heat Shock Protein Chaperone Interaction Network as Guardian of the Proteome in Health and Disease. Current Immunology Reviews. 2017;13(1):23. DOI: $10.2174 / 157339551301170906115238$

11. Gomez-Pastor R, Burchfiel ET, Thiele DJ. Regulation of heat shock transcription factors and their roles in physiology and disease. Nature Reviews Molecular Cell Biology. 2017. DOI: 10.1038/nrm.2017.73

12. Lu XY, Chen L, Cai XL, Yang HT. Overexpression of heat shock protein 27 protects against ischaemia/ reperfusion-induced cardiac dysfunction via stabilization of troponin I and T. Cardiovascular research. 2008;79(3):500-8. DOI: 10.1093/cvr/cvn091

13. Shams S, Shafi S, Bodman-Smith K, Williams P, Mehta S, Ferns GA. Anti-heat shock protein-27 (Hsp-27) antibody levels in patients with chest pain: association with established cardiovascular risk factors. Clinica chimica acta; international journal of clinical chemistry. 
2008;395(1-2):42-6.

14. Ghayour-Mobarhan M, Sahebkar A, Parizadeh SM, Moohebati M, Tavallaie S, Rezakazemi-Bajestani SM, et al. Antibody titres to heat shock protein 27 are elevated in patients with acute coronary syndrome. International journal of experimental pathology. 2008;89(3):209-15. DOI: 10.1111/j.1365-2613.2008.00586.x

15. Pourghadamyari H, Moohebati M, Parizadeh SM, Falsoleiman H, Dehghani M, Fazlinezhad A, et al. Serum antibody titers against heat shock protein 27 are associated with the severity of coronary artery disease. Cell stress \& chaperones. 2011;16(3):309-16. DOI: 10.1007/s12192-010-0241-7

16. Kuang H-J, Zhao G-J, Chen W-J, Zhang M, Zeng G-F, Zheng X-L, et al. Hsp27 promotes ABCA1 expression and cholesterol efflux through the PI3K/ PKC $/$ Sp1 pathway in THP-1 macrophages. European Journal of Pharmacology. 2017. DOI: 10.1016/j.ejphar.2017.06.015

17. Shi C, Chen Y-X, Diao C, Batulan Z, OBrien ER. Novel Atheroprotection Therapy: Reduction in Serum Lipid Levels by HSP27 Via Down-regulation of HNF1a and PCSK9 Expression. Am Heart Assoc; 2016.

18. Singh T, Newman AB. Inflammatory markers in population studies of aging. Ageing research reviews. 2011;10(3):319-29. DOI: 10.1016/j.arr.2010.11.002

19. Ghayour-Mobarhan M, Moohebati M, Esmaily H, Ebrahimi M, Parizadeh SMR, Heidari-Bakavoli AR, et al. Mashhad stroke and heart atherosclerotic disorder (MASHAD) study: design, baseline characteristics and 10-year cardiovascular risk estimation. International journal of public health. 2015;60(5):561-72. DOI: 10.1007/s00038-015-0679-6

20. Tremblay AJ, Morrissette H, Gagné J-M, Bergeron J, Gagné C, Couture P. Validation of the Friedewald formula for the determination of low-density lipoprotein cholesterol compared with $\beta$-quantification in a large population. Clinical biochemistry. 2004;37(9):785-90. DOI: 10.1016/j.clinbiochem.2004.03.008

21. Austin PC. A comparison of 12 algorithms for matching on the propensity score. Statistics in Medicine. 2014;33(6):1057-69. DOI: 10.1002/sim.6004

22. Ghayour-Mobarhan M, Rahsepar A, Tavallaie S, Rahsepar S, Ferns G. The potential role of heat shock proteins in cardiovascular disease: evidence from in vitro and in vivo studies. Advances in Clinical Chemistry. 2009;48:27-72. DOI: 10.1016/S0065-2423(09)48002-8

23. Kim J, Yenari M. Heat Shock proteins and the Stress Response. Primer on Cerebrovascular Diseases. 2017:273. DOI: 10.1016/B978-0-12-803058-5.00056-4
24. Abulafia-Lapid R, Elias D, Raz I, Keren-Zur Y, Atlan $\mathrm{H}$, Cohen IR. T cell proliferative responses of type 1 diabetes patients and healthy individuals to human hsp60 and its peptides. Journal of autoimmunity. 1999;12(2):121-9. DOI: 10.1006/jaut.1998.0262

25. Ghayour-Mobarhan M, Sahebkar A, Parizadeh SMR, Moohebati M, Tavallaie S, RezaKazemi-Bajestani SM, et al. Antibody titres to heat shock protein 27 are elevated in patients with acute coronary syndrome. International journal of experimental pathology. 2008;89(3):209-15. DOI: $10.1111 / \mathrm{j} .1365-2613.2008 .00586 . \mathrm{x}$

26. Kargari M, Tavassoli S, Avan A, Ebrahimi M, Azarpazhooh MR, Asoodeh R, et al. Relationship between serum anti-heat shock protein 27 antibody levels and obesity. Clinical Biochemistry. 2017. DOI: 10.1016/j.clinbiochem.2017.02.015

27. Viollet B, Guigas B, Garcia NS, Leclerc J, Foretz M, Andreelli F. Cellular and molecular mechanisms of metformin: an overview. Clinical science. 2012;122(6):253-70. DOI: 10.1042/CS20110386

28. Dai S, Tang Z, Cao J, Zhou W, Li H, Sampson S, et al. Suppression of the HSF1-mediated proteotoxic stress response by the metabolic stress sensor AMPK. The EMBO journal. 2015;34(3):275-93. DOI: 10.15252/ embj.201489062

29. Ghayour-Mobarhan M, Lamb DJ, Vaidya N, Livingstone C, Wang T, Ferns GA. Heat shock protein antibody titers are reduced by statin therapy in dyslipidemic subjects: a pilot study. Angiology. 2005;56(1):61-8. DOI: 10.1177/000331970505600108

30. Moohebati M, Bidmeshgi S, Azarpazhooh MR, Daloee MH, Ghayour-Mobarhan M, Tavallaie S, et al. Simvastatin treatment reduces heat shock protein 60,65 , and 70 antibody titers in dyslipidemic patients: A randomized, double-blind, placebo-controlled, cross-over trial. Clin Biochem. 2011;44(2-3):192-7. DOI: 10.1016/j. clinbiochem.2010.09.016

31. Aryanpour R, Parizadeh SMR, Moohebati M, Tavallaie S, Sahebkar AH, Mohammadi S, et al. SimvastatinTreatment is not Associated with Changes in Serum Concentrations of Heat ShockProteins -60 and -70 in Patients with Dyslipidemia. Pharmaceutical Sciences.20(2):46-51.

32. Burut DFP, Borai A, Livingstone C, Ferns G. Serum heat shock protein 27 antigen and antibody levels appear to be related to the macrovascular complications associated with insulin resistance: a pilot study. Cell Stress and Chaperones. 2010;15(4):379-86. DOI: 10.1007/s12192-009-0152-7 